\title{
Value of three-dimensional computed tomography reconstruction in the treatment of posterior tibial plateau fractures
}

\author{
J. Van den Berg a,1, B. Struelensa,1, S. Nijsa,b, H. Hoekstra a,b,* \\ a University Hospitals Leuven, Department of Trauma Surgery, B-3000 Leuven, Belgium \\ b KU Leuven - University of Leuven, Department of Development and Regeneration, B-3000 Leuven, Belgium \\ * Corresponding author at: University Hospitals Leuven, Department of Trauma Surgery, Herestraat 49, B-3000 Leuven, Belgium. \\ E-mail address: harm.hoekstra@uzleuven.be. (H. Hoekstra). \\ 1 These authors contributed equally to this work.
}

Keywords: Knee, Tibial plateau fracture, Posterior column, Classification, 3D reconstruction

\begin{abstract}
Background: Indication for surgical treatment of posterior tibial plateau fractures (TPFs) remains up for debate. Threedimensional computed tomography (3D-CT) reconstruction can provide insight into fracture morphology and could improve treatment strategy and surgical planning. In this study, we investigated the value of 3D-CT reconstruction in the treatment of posterior TPF and evaluated the influence on surgical decision-making.

Methods: CT images of 34 cases with a TPF involving the posterior column were included and digitally presented to a panel of five international observers at two intervals. At the first evaluation, only coronal, axial and sagittal images were shown. After an interval of at least three weeks, 3D-CT reconstruction images were added. During both surveys, observers were asked to classify the TPF according to the revised three-column classification (rTCC), as well as to define operative strategy.

Results: When using 2D images, overall multirated kappa value was 0.48 , with an average pairwise agreement of $68 \%$. After adding 3D images, overall multirated kappa value was 0.43 , with average pairwise agreement of $67 \%$. Hierarchical logistic regression of decision to operate on image condition (3D vs. 2D) shows an odds ratio of 2.01 (95\% confidence interval, $1.11-$ 3.67), $P=.022$. Increase in operative indication was seen mainly in posterolateral fractures.

Conclusion: This study investigated the value of 3D classification in the treatment of posterior column TPF. Contrary to expectations, the addition of 3D images to the assessment did not reduce but rather appeared to increase operative indications, especially in fractures involving the posterolateral region.
\end{abstract}

\section{Introduction}

To date, the outcome of tibial plateau fractures (TPFs) remains uncertain. Recently, fractures of the posterior surface of the tibial plateau have been identified as an important prognostic factor on clinical outcome [1]. In contrast to commonly used classification systems (Schatzker, AO/OTA), posterior TPFs are better depicted according to the three-column concept [1-6]. According to the three-column concept, every column fracture (articular depression in combination with a cortical interruption) should be treated surgically [7]. TPFs that were treated in accordance with this concept reported a better functional outcome $[1,7]$. However, there seems to be a flaw in the three-column concept. Fracture patterns of multiple-column fractures are more comprehensive than can be adequately expressed by three-column classification (TCC) $[1,5,8,9]$. Fracture morphology and the risk for secondary displacement are case specific and although thoroughly investigated with computed tomography (CT) images, no current classification seems to fully satisfy. Case selection for posterior fixation is still highly debated and our current evidence cannot sufficiently direct our decision-making.

Recently, three-dimensional (3D) classification for calcaneal fractures has been shown to be a reliable tool to gain more insight into fracture morphology and thus to improve treatment strategy [10]. According to the literature, additional 3D images provide insights in several other orthopedic domains as well [11,12]. In 2009, Hu et al. suggested 3D-CT images to be a more reliable radiographic modality than 2D-CT in evaluation of fracture patterns in TPFs [13]. CT imaging in these fractures is widely accepted as the gold standard in preoperative investigation and planning and recently 3D reconstruction is more widely available. However, the impact of adding 3D-reconstruction on preoperative planning and decision-making has not been thoroughly evaluated. Therefore, the goal of the present study was to determine the value of $3 \mathrm{D}$ reconstruction in classification and treatment of TPFs with posterior involvement. We assessed the intra-observer and interobserver variability between 2D and 3D imaging and evaluated the influence of 3D images on surgical decision-making and clinical practice. 


\section{Patients \& methods}

This study was completed in compliance with national legislation and the guidelines of the ethics committee of the University Hospitals Leuven. CT images of 34 patients with a TPF involving the posterior column treated between 24 July 2014 and 29 May 2018 were included. Selection of cases was based on quality of imaging series providing highquality 3D reconstruction. All CT images were recorded using the Aquilion One Volume CT scanner (Toshiba, Tokyo, Japan), with a table pitch of 0.641 , collimation of $0.5 \times 64$, slice thickness of $0.5 \mathrm{~mm}$, slice increment of $0.5 \mathrm{~mm}$, and rotation time of $0.5 \mathrm{~s}$. All images were anonymized and digitally presented to a panel of five international observers to classify the TPFs (all posterior column fractures, possibly combined with a medial and/or lateral column fracture). The questionnaire and the images were presented on a secure, custom-made website (https://rtcc.lifehost.org). The reference drawing of the revised three-column classification (rTCC) was presented during the survey. At the first evaluation, the 2D-CT images (coronal, axial and sagittal) were shown. After a minimum interval of three weeks, in addition, 3D reconstructed images were presented to the observers. During both surveys, the observers were asked to classify the TPFs according to the rTCC, as well as to define the operative strategy. In this study, both intra- and interobserver reliability were examined.

\subsection{The three-column concept and rTCC}

The rTCC is shown in Figure 1. According to the three-column concept as proposed by Luo et al. in 2010, an independent column fracture is defined as an articular depression with a fracture of the column wall [2]. Subsequently, column fractures should be fixated accordingly [7]. According to rTCC classification, lateral column fractures that extend into the posterolateral corner (dotted area, OCD), are defined as extended lateral column fractures (OAD). Posterolateral corner fractures extending medially of the fibular head are referred to as posterolateral column fractures (OEC) and should be treated from posterior. The posterior column (OCB) can be divided on the midline in a posterolateral (OEC) and posteromedial column (OEB).

\subsection{Statistical analysis}

Using the kappa statistic, the reliability of the fracture classification made by the same observer on separate occasions (intraobserver reliability) or by different observers on the same occasion (interobserver reliability) was measured. The reliability guidelines were scored using the Landis and Koch guidelines [15]. The distribution of the rTCC classification scores was compared between the 2D-CT images and the 3D-CT images. First, we recorded the percentage of agreement, which equals the probability that two observers would give the same score. Second, the Fleiss kappa ( $\mathrm{k}$ ) for multiple observer was recorded. The Fleiss $\mathrm{K}$ is a measure of the degree of interobserver agreement beyond the level of chance. Next, an analysis of decision to operate based on 3D-CT vs. 2D-CT images using hierarchical logistic regression was carried out. The analyses were performed using SPSS Statistics for Windows, version 25.0 (SPSS Inc., Chicago, IL, USA).

\section{Results}

A total of 34 multislice CT-scans of patients with a TPF with posterior involvement according to rTCC classification were assessed by a panel of five international observers. The average interval between surveys was 7.5 weeks (range: 3.1-10.3). The intra-observer agreement and reliability between 2D and 3D classification was, on average, $72.4 \%$ (range: $56-91 \%$ ) with an average Kappa of 0.53 (range: 0.17-0.85) (Table 1). A hierarchical logistic regression of the decision to operate on image condition (3D vs. 2D): OR of image condition was $2.01(95 \% \mathrm{Cl}, 1.11-3.67), \mathrm{P}=$ .022 (Table 2). The average pairwise interobserver agreement was $68 \%$ with an overall multirated kappa value of 0.48 when using $2 \mathrm{D}$ images. Adding the $3 \mathrm{D}-\mathrm{CT}$ images during the second survey resulted in an overall multirated kappa value of 0.43 , with an average pairwise agreement of $67 \%$ (Table 3 ).

All 13 cases where an increase in operative indication was observed were separately assessed for fracture morphology. In one case, there was a posteromedial fracture, and in 12 cases, both the posterior and lateral columns were involved. Of these 12 cases, 10 cases involved the posterolateral column exclusively (Figure 1, OEC). 


\section{Discussion}

Because no current comprehensive classification system for the treatment of posterior TPF is available, the goal of this study was to determine the added value of 3D reconstruction in surgical decision-making and treatment of TPFs with posterior involvement. Firstly, we assessed the intra- and interobserver variability of rTCC classification between 2D and 3D imaging. Secondly, we evaluated the influence of added 3D imaging on surgical decision-making and clinical practice.

On average, intra-observer agreement was moderate on classification with and without 3D imaging available [14]. Although, a wide range in intra-observer agreement was found between observers $(0.17-0.85)$. The interobserver agreement on classification decreased slightly when 3D was added with kappa values of 0.48 and 0.43 , respectively. The pairwise average interobserver agreement before 3D and after, was $68 \%$ and $67 \%$, respectively. Regarding decision to operate, we found an increase of $8.2 \%$ in favor of fixation of posterior column fixation when assessing $3 \mathrm{D}$ reconstructed images with an odds ratio of $2.01(\mathrm{P}=.022)$. This is in contrast to the findings of other studies, reporting an overestimation of comminution based on 2D images in fractures of the foot, and therefore less need for surgery $[10,15]$. Although all observers show an increase in operative indication, the results are highly influenced by observers 4 and 5 as shown by their low intra-observer agreement ( 0.46 and 0.17 , respectively).

In the current literature, conflicting evidence exists concerning the added value of 3D-CT images in fracture management. Recently, Misselyn et al. demonstrated an improved interobserver agreement in fracture classification when adding 3D-CT images regarding calcaneal fractures [10]. Specifically, in proximal tibia fractures, CT-based classifications have been shown to be a reliable tool to gain more insight into fracture morphology and thus improve treatment strategy $[4,8]$. In a recent systematic review, Millar et al. demonstrated an interobserver kappa value ranging from 0.37 to 0.73 when using the Schatzker classification [16]. Castiglia et al. reported an interobserver reliability of 0.64 for the Schatzker classification with the use of 3D-CT images, and, they showed that the use of CT (compared to a radiograph) also had an impact on the surgical approach. Furthermore, they reported an improvement in the interobserver reliability with added CT imaging. However, during each assessment the observers were shown all imaging techniques sequentially. Although a decisive impact in surgical decision was shown in TPFs with added CT imaging, no differences were reported after adding 3D imaging [17]. Nevertheless, the results of the comparison between the assessments at different times, are not reported. In our current study, the 3D imaging was only presented to the observers after the minimum time interval of three weeks.

In the series presented by Zhu et al., there was a 'substantial interobserver agreement' with the use of the TCC based on 2D-CT images [4]. Our current study showed only moderate interobserver agreement. However, this may partially be explained by the classification system, as the TCC only has four categories (including zero column fractures), while the rTCC has an additional class [6]. In 2015, Mellema demonstrated that the addition of 3D-CT reconstructions did not improve the interobserver reliability of CT-based evaluation of TPFs when using the Schatzker or Luo's TCC [3]. Although our results on interobserver agreement for the rTCC are in line with Mellema et al., the current findings show an increase in surgical management of posterior TPFs (odds ratio 2.01, $\mathrm{P}=.022$ ). This suggests an inherent failure of current 2D-CT classification on guiding surgical treatment. A recent study by Pätzold et al. suggests a new classification scheme based on the 3D geometry of bicondylar proximal tibial fractures guided by the direction of fracture lines. They report a kappa value of 0.936 for the 3D classification scheme compared with 0.720 and 0.785 for the AO/OTA and the Schatzker classification, respectively [18].

Optimal treatment strategies for TPF with posterior involvement are not well established and decision-making is mostly based on degree of articular depression, fracture morphology and patient characterization. Recently, there has been increased interest in the direction of fracture lines towards treatment planning. 3D-CT visualization can improve understanding of fracture patterns. Moreover, visualization of posterior involvement with specific coronal fracture lines may improve surgical decision-making $[5,18]$. Current literature, including frequently referenced Luo et al. and Wang et al. does not provide absolute criteria or values for posterior plate osteosynthesis. The finding of increased operative indication in this study after review of 3D reconstruction suggests a limitation of current classification schemes. As previously shown by different authors, TPF patterns are not always well presented by TCC $[8,18,19]$. Furthermore, we have shown that decision-making in fractures of both the posterolateral column (Figure $1, \mathrm{OEC}$ ) and extended lateral column (Figure 1, OAD) are influenced by 3D-CT reconstruction (Figure 1). In Figure 2, we present an illustrative case wherein the opinion of the observer panel changed to posterior operative indication. 
This further substantiates the hiatus of current classifications and the ongoing discussion regarding treatment strategies of posterolateral TPFs.

In this study, we did not investigate the correlation between the increased operative indication and functional outcome. Therefore, no definite conclusions can be drawn towards future guiding of surgical planning and management of posterior TPFs. An increase in operative indications could potentially harm patient outcome. Therefore, more research is needed to verify optimal indication for posterior fixation of these fractures. Another weakness of this study was the limited number of experienced knee trauma surgeons involved, as only five observers were included. Therefore, the largest impact on the operative indication is based on the observers with the lowest intra-observer reliability.

\section{Conclusion}

The current findings indicate that utilization of 3D-CT images in preoperative planning might increase surgical intervention on posterior column TPFs, especially in fractures involving the posterolateral column. To our knowledge, the current study is the first to assess the added value of 3D-reconstruction in rTCC classification and towards surgical decision-making in posterior fractures. Further investigation is necessary with a focus on specific fracture patterns and morphology towards guidance in surgical decision-making.

Declaration of competing interest

The authors have no conflicts of interest to declare. This research did not receive any specific grant from funding agencies in the public, commercial, or not-for-profit sectors.

Acknowledgments

The authors acknowledge Walter Coudyzer (Department of Radiology, University Hospitals Leuven, Leuven, Belgium) and all the observers who participated in the present study.

\section{References}

[1] Van Den Berg J, Reul M, Cardozo MN, Starovoyt A, Geusens E, Nijs S, et al. Functional outcome of intra-articular tibial plateau fractures: the impact of posterior column fractures; $2017 ; 1865-73$.

[2] Luo C-F, Sun H, Zhang B, Zeng B-F. Three-column fixation for complex tibial plateau fractures. J Orthop Trauma 2010;24:683-92. https://doi.org/10.1097/BOT. 0b013e3181d436f3.

[3] Mellema JJ, Doornberg JN, Molenaars RJ, Ring D, Kloen P, et al. Interobserver reliability of the Schatzker and Luo classification systems for tibial plateau fractures. Injury 2016;47:944-9.

[4] Zhu Y, Yang G, Luo C, Smith WR, Hu C, Gao H, et al. Computed tomography-based Three-Column Classification and assessment of its reproducibility. J Trauma Acute Care Surg 2012;73:731-7.

[5] Kim CW, Lee CR, An KC, Gwak HC, Kim JH, Wang L, et al. Predictors of reduction loss in tibial plateau fracture surgery: focusing on posterior coronal fractures. Injury 2016;7:1483-7.

[6] Hoekstra H, Kempenaers K, Nijs S. A revised 3-column classification approach for the surgical planning of extended lateral tibial plateau fractures. Eur J Trauma Emerg Surg 2017;43:637-43.

https://doi.org/10.1007/s00068-016-0696-z.

[7] Wang Y, Luo C, Zhu Y, Zhai Q, Zhan Y, QiuW, et al. Updated Three-column Concept in surgical treatment for tibial plateau fractures - a prospective cohort study of 287 patients. Injury 2016;47:1488-96.

[8] Molenaars R,Mellema J, Doornberg J, Kloen P. Tibial plateau fracture characteristics: computed tomography mapping of lateral,medial, and bicondylar fractures. J Bone Joint Surg Am 2015;97-A:1512-20.

[9] Yao X, Xu Y, Yuan J, Lv B, Fu X,Wang L, et al. Classification of tibia plateau fracture according to the "fourcolumn and nine-segment". Injury 2018;49:2275-83.

[10] Misselyn D, Nijs S, Fieuws S, Shaheen E, Schepers T. The journal of foot \& ankle surgery improved interobserver reliability of the Sanders classification in calcaneal fractures using segmented three-dimensional prints. J Foot Ankle Surg 2018;57:440-4 Available from: https://doi.org/10.1053/j.jfas.2017.10.014.

[11] Jacquot A, Poussange N, Charrissoux J, Clavert P, Obert L, Pidhorz L. Usefulness and reliability of two- and three-dimensional computed tomography in patients older than 65 years with distal humerus fractures. Orthop Traumatol Surg Res 2014;100:275-80 Available from: https://doi.org/10.1016/j.otsr.2014.01.003. 
[12] Sebaaly A, Riouallon G, Zaraa M, Upex P, Marteau V, Jouffroy P. Standardized three dimensional computerised tomography scanner reconstructions increase the accuracy of acetabular fracture classification. Int Orthop 2018;42:1957-65.

[13] Hu Y, Ye F, Ji A, Qiao G, Liu H. Three-dimensional computed tomography imaging increases the reliability of classification systems for tibial plateau fractures. Injury 2009;40:1282-5.

[14] Landis J, Koch G. The measurement of observer agreement for categorical data. Biometrics 1977:159-74.

[15] Allon S, Mears D. Three dimensional analysis of calcaneal fractures. Foot Ankle Int 1991;11:254-63.

[16] Millar SC, Arnold JB, Thewlis D, Fraysse F, Solomon LB. A systematic literature review of tibial plateau fractures: what classifications are used and how reliable and useful are they? Injury 2018;49:473-90 Available from: https://doi.org/10.1016/j.injury.2018.01.025.

[17] Castiglia M, Nogueira-Barbosa M, Messias A, Salim R, Fogagnolo F, Schatzker J, et al. The impact of computed tomography on decision making in tibial plateau fractures. J Knee Surg 2018;31(10):1007-14.

https://doi.org/10.1055/s-0038-1627464.

[18] Pätzold R, Friederichs J, von Rüden C, Panzer S, Bühren V, Augat P, et al. The pivotal role of the coronal fracture line for a new three-dimensional CT-based fracture classification of bicondylar proximal tibial fractures. Injury 2017;48:2214-20.

[19] Krause M, Preiss A, Müller G, Madert J, Fehske K, Neumann MV, et al. Intra-articular tibial plateau fracture characteristics according to the "Ten segment classification". Injury 2016;47:2551-7.

https://doi.org/10.1016/j.injury.2016.09.014. 


\section{Figures}

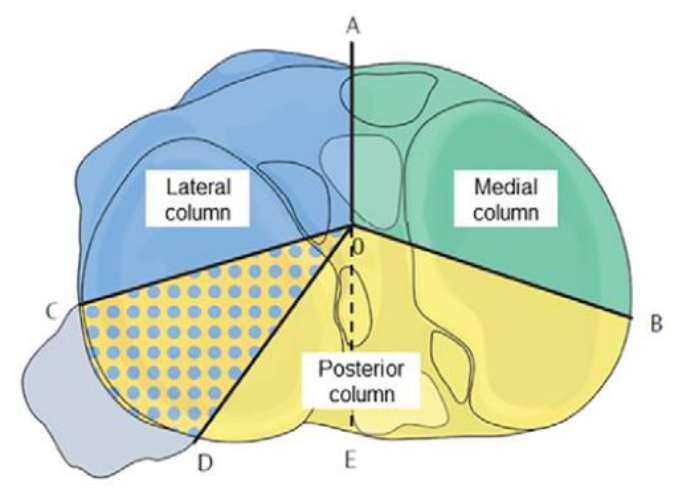

Figure 1. The revised three-column classification. Lateral column fractures that extend into the posterolateral corner (dotted area, OED), are defined as extended lateral column fractures (OAD). Posterolateral corner fractures extending medially of the fibular head are referred to as posterolateral column fractures (OEC).

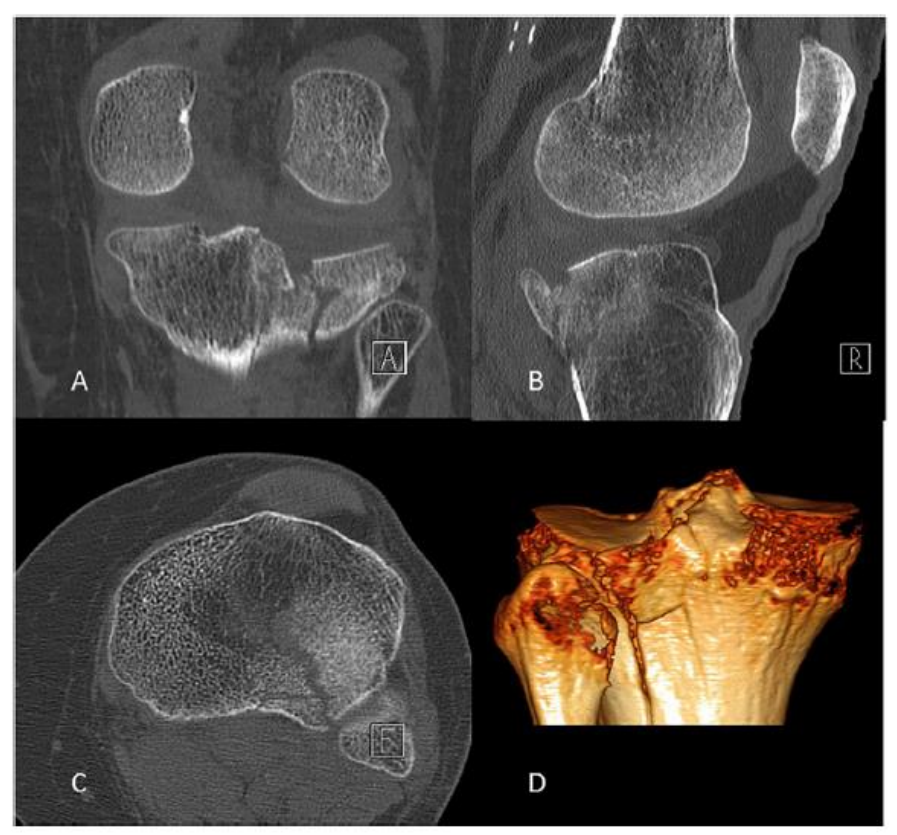

Figure 2. Illustrative case of a 65-year-old woman, who sustained a tibial plateau fracture after a fall in a hole at road works. Coronal view (a), sagittal view (b), axial view (c) and three-dimensional computed tomography (3D-CT) reconstruction are presented. Preoperative imaging shows a lateral column fracture combined with posterior column fracture (posterolateral column, $\mathrm{OEC}$ ). The coronal and axial views show the fracture line oriented in the sagittal plane, which can be treated via lateral approach $(a, c)$. Axial and sagittal views show a separate fracture component with fracture line in the coronal plane (b, c). 3D-CT reconstruction shows the posterior aspect of the proximal tibia with both the sagittal fracture line and the posterior fragment in view. 
Tables

Table 1: Intra-observer agreement and reliability between two-dimensional (2D) and three-dimensional (3D) classification.

\begin{tabular}{lll}
\hline & Agreement & Kappa (intra-observer) \\
& 2D-3D classification & 0.52 \\
\hline Observer 1 & $23(68 \%)$ & 0.85 \\
Observer 2 & $31(91 \%)$ & 0.65 \\
Observer 3 & $27(79 \%)$ & 0.46 \\
Observer 4 & $23(68 \%)$ & 0.17 \\
Observer 5 & $19(56 \%)$ & \\
\hline
\end{tabular}

Table 2: Analysis of decision to operate based on three-dimensional (3D) vs. two-dimensional (2D) images.

\begin{tabular}{llll}
\hline & 2D operate, $\mathrm{n}(\%)$ & 3D operate, $\mathrm{n}(\%)$ & Difference (95\% confidence interval) \\
\hline Observer 1 & $20(59 \%)$ & $22(65 \%)$ & $6(-13,24)$ \\
Observer 2 & $19(56 \%)$ & $21(62 \%)$ & $6(-7,18)$ \\
Observer 3 & $19(56 \%)$ & $20(59 \%)$ & $3(-5,11)$ \\
Observer 4 & $16(47 \%)$ & $21(62 \%)$ & $15(-1,29)$ \\
Observer 5 & $25(74 \%)$ & $29(85 \%)$ & $12(-3,27)$ \\
\hline
\end{tabular}

Table 3 Interobserver agreement and reliability when using two-dimensional (2D) or three-dimensional (3D) images.

\begin{tabular}{llll}
\hline 2D/3D & Observer 1 & Observer 2 & Observer 3 \\
\hline Observer 1 & & & \\
Observer 2 & $28(82 \%) / 21(62 \%)$ & $29(85 \%) / 28(82 \%)$ & \\
Observer 3 & $27(79 \%) / 26(76 \%)$ & $29(85 \%) / 26(76 \%)$ & $24(71 \%) / 24(71 \%)$ \\
Observer 4 & $25(74 \%) / 21(62 \%)$ & $19(56 \%) / 20(59 \%)$ & $14(41 \%) / 23(68 \%)$ \\
Observer 5 & $15(44 \%) / 17(50 \%)$ & & $22(65 \%) / 22(65 \%)$ \\
\hline
\end{tabular}

